\title{
Acute Respiratory Distress Revealing an Unrecognized Tracheostomy Cannula at the Bronchial Level in the Pandemic COVID Era
}

\author{
Mohamed Chehbouni and Othmane Benhoummad
}

\section{ABSTRACT}

The tracheostomy is a surgical opening of the trachea which is made at the level of its anterior face, $2 \mathrm{~cm}$ above the sternal fork permitting the placement of a tracheostomy cannula in a temporary or definitive way and this according to the indication of its realization. The tracheostomy is the surgical connection of the trachea to the skin. The prolonged wearing of a tracheostomy cannula at the level of the tracheostomy orifice can expose to certain complications which can prove to be serious. It presents risks of postoperative complications, especially late ones. The most frequent are granulomas, tracheal stenosis, infections, and fistulas. Tracheal or tracheobronchial migration of the tracheostomy cannula remains exceptional. We present a very rare case of an elderly subject followed for Parkinson's disease with a dementia syndrome, who underwent a total laryngectomy 3 years ago with prolonged placement of a tracheostomy cannula at the tracheostomy orifice. The patient presented to the emergency room with progressively worsening respiratory distress and desaturation, evolving in a febrile context, suggesting a sars cov19 infection in the current epidemiological context. A thoracic CT scan rectified the diagnosis by showing a pneumopathy on an unrecognized tracheotomy cannula at the tracheobronchial level. Indeed, this complication is secondary to the embrittlement of the cannula following poor maintenance. The use of a tracheostomy cannula for a long period of time requires a regular and particular surveillance, a rigorous maintenance and care of the cannula to avoid the occurrence of complications which can be serious.

Keywords: Tracheostomy tube, rigid bronchoscopy, Tracheobronchial tree, Foreign body.

\section{INTRODUCTION}

The prolonged wearing of a tracheostomy cannula through the tracheostomy or tracheostomy orifice allows short-circuiting the supraglottic upper airway [1]. Its indications are multiple, and its complications described in the literature are the same. The acute complications are hemorrhage, pneumothorax, obstruction of the tracheostomy tube and wound infection. Late complications include granuloma formation, tracheal stenosis, innominate artery ulceration, and tracheoesophageal fistula [2]. Fracture and migration of the tracheostomy cannula into the tracheobronchus remains a rare complication, even more so if it is not recognized.

\section{OBSERVATION}

The patient was 70 years old, followed for a Parkinson's disease with a dementia syndrome, laryngectomized 3 years ago for a laryngeal cancer, wearing a metallic tracheostomy cannula at the tracheostomy orifice. The patient was admitted to the emergency room with progressively stump bronchus.
Submitted : June 06, 2021

Published : June 29, 2021

ISSN: 2593-8339

DOI: $10.24018 /$ ejmed.2021.3.3.923

Mohamed Chehbouni *

Specialist in ENT and Cervico-Facial Surgery, Provincial Hospital Mokhtar Soussi, Taroudant, Morocco.

(e-mail: dr.chehbouni gmail.com) Othmane Benhoummad

Assistant professor in ENT and Cervico-Facial Surgery, Agadir University Hospital Center, Ibn Zohr University, Agadir, Morocco. (e-mail: benhoummadorl gmail.com).

*Corresponding Author

worsening respiratory distress evolving for 4 days in a context of uncalculated fever. On examination, the patient was conscious, hemodynamically stable, febrile at $38.3^{\circ}$ and moderately dyspneic with mild signs of respiratory distress.

Given the current epidemiological context of the covid pandemic and this clinical picture, the patient was redirected to the special covid circuit. A thoracic CT scan performed in emergency allowed to rectify the diagnosis by showing a right basi-pulmonary alveolar syndrome on an unrecognized cannula migrating into the left tracheo-bronchial (Fig. 1).

The patient had two tracheostomy cannulas, one at the tracheostomy port and one at the tracheobronchial level.

Indeed, it went into the left stump bronchus and allowed pulmonary ventilation on that side but obstructed the right

On further questioning with his family, his relatives reported the disappearance of the inner lining of a tracheostomy cannula 10 days ago without being able to think of its fracture and its migration into the tracheobronchial. This is due to the loss of contact of the patient who cannot express himself correctly because of the dementia syndrome for which he has not been followed up. 


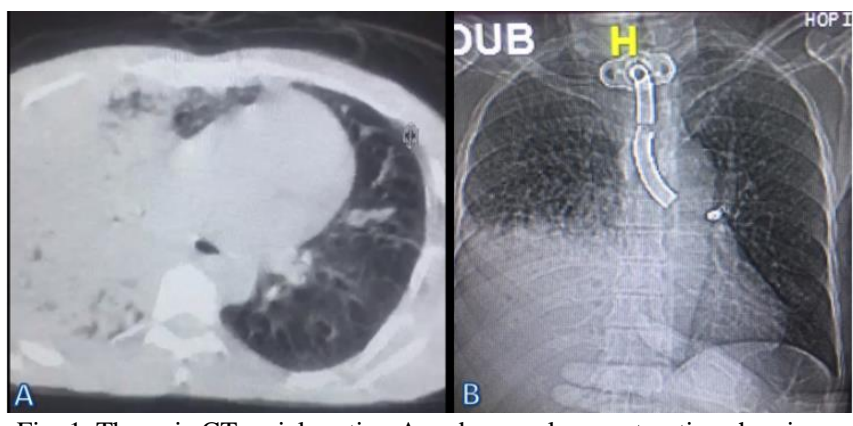

Fig. 1. Thoracic CT: axial section A and coronal reconstruction showing a right basipulmonary alveolar syndrome complicating a foreign body tracheotomy cannula - unrecognized in left tracheobronchial.

The extraction was performed with a rigid bronchoscope introduced through the tracheostomy orifice under general anesthesia after venous antibiotic therapy with amoxicillin clavulanic acid. It turned out that the internal lining of the tracheostomy cannula was fractured at its external end and that it remained permeable and not obstructed by mucous secretions.

\section{DISCUSSION}

Tracheostomy is one of the most frequently performed procedures in head and neck surgery. It is considered a safe procedure to ensure adequate ventilation for a patient with an obstructed airway or in need of long-term ventilation. However, prolonged wearing of the tracheostomy cannula may be responsible for late complications such as tracheostomal stenosis, granulation tissue formation, brachiocephalic trunk ulceration, tracheoesophageal and tracheoinnominated fistulas, and tracheomalacia [3]-[7]. Tracheostomy cannula fracture and migration into the tracheobronchial tree is a rare complication, often revealed by asthma, chronic bronchitis or pneumonia. This complication was first described in 1960 by Bassoe and Boe [2]-[8]. And since then, this rare type of complication has been reported in the literature with different types of tracheostomy cannula. Indeed, the design of a metal-based tracheostomy cannula allowed for its prolonged wear due to its physical properties that make it unlikely to fracture or be damaged by contact with the human body or during cleaning processes. Therefore, a metal cannula was thought to be appropriate for prolonged wear [9]. Initially, silver, steel, copper, or zinc were the raw materials for the manufacture of these tracheostomy cannulas, but they have been found to be corrodible by tracheal secretions and repeated boiling [10]. Therefore, stainless steel is currently the primary material for tracheostomy cannulas due to its resistance to chemical corrosion. However, fracture of a stainless steel tracheostomy cannula has occasionally been reported in the literature [2]-[11].

The fracture of the tracheostomy tube can occur from the 5 th day of its placement as well as 22 years later [3]. In our case it occurred three years later. The weak areas of the tracheostomy cannula where this fracture can occur are at the junctions; either between the tube and the cervical plate, or between the distal end of the tube and the fenestration site [12]-[17]. Contrary to the literature, the fracture was located at the tip of the inner liner of the cannula.
Several causes of cannula fracture have been proposed: design and manufacturing defects, internal mechanical stresses on the surface, corrosion by alkaline bronchial secretions, exposure to the atmosphere, disinfectant fluids (sodium hypochlorite solution), repeated cleaning/boiling or sterilization, repeated removal and reinsertion, and tube aging [9], [10], [18]-[20] .

Once the tracheostomy cannula is fractured, it often migrates to the right stem bronchus for anatomical reasons [9]. Paradoxically, in our patient, the cannula migrated to the left tracheobronchial.

As with any tracheobronchial foreign body, the telltale signs can be acute or chronic. They may include cough, hemoptysis, wheezing, recurrent pneumonia, or respiratory distress. As it may manifest itself as difficulty in aspiration or re-insertion of the inner tube, or any change in the tube [9], [21]. In our case, tracheostomy tube fracture may be due to prolonged use, lack of periodic inspection and aging of the tube.

To overcome this type of complication, some recommendations are suggested:

A change of the tracheostomy cannula every 6 months, daily or twice daily cleaning of the inner liner of the tracheostomy cannula with a change of the tracheostomy port dressing on a daily basis. A weekly change of the cannula attachments. Patient use of two tracheostomy cannulas alternately to reduce wear and tear. Establishment of a monitoring and control system by each hospital involved in the management of tracheostomized patients. Information and training of the nursing staff as well as the patients about the different complications that can occur in a tracheostomized patient. In case of emergency, immediate contact with the hospital and a good referral system are essential for early detection and management of these complications [11], [15], [20].

\section{CONCLUSION}

The fracture and migration of a tracheostomy cannula into the tracheobronchus is a rare and preventable complication. It should be taken into account in the event of respiratory distress in a patient with a tracheostomy cannula. All the more so if the patient has a neurological pathology limiting contact and expression. Educating the patient's family and primary caregivers about the method of cleaning the tracheostomy tube and the various possible complications can prevent this complication.

\section{REFERENCES}

[1] Chouikh, C. et al. Migration trachéale d'une canule de trachéotomie: complication exceptionnelle. Pan Afr Med J 18, (2014).

[2] Piromchai, P., Lertchanaruengrit, P., Vatanasapt, P., Ratanaanekchai, T. \& Thanaviratananich, S. Fractured metallic tracheostomy tube in a child: a case report and review of the literature. J Med Case Reports 4 , 234 (2010).

[3] Fractured tracheostomy tube migrating into the tracheobronchial tree a rare complication - PubMed. https://pubmed.ncbi.nlm.nih.gov/21545073/.

[4] Streitz, J. M. \& Shapshay, S. M. Airway Injury after Tracheotomy and Endotracheal Intubation. Surgical Clinics of North America 71, 1211-1230 (1991).

[5] Pawar. A Rare Early Complication of Tracheostomy. OMJ 26, 48-49 (2011). 
[6] Kashoob, M., Al Washahi, M. \& Tandon, R. Aspiration Pneumonia Due to Migration of Fracture Tracheostomy Tube after 14 Years of Use. Oman Med J 35, e113-e113 (2020).

[7] Epstein, S. K. Late Complications of Tracheostomy. Respiratory Care 50,8 (2005).

[8] Bassøe, H. H. \& Bøe, Johs. Broken tracheotomy tube as a foreign body. The Lancet 275, 1006-1007 (1960).

[9] So-ngern, A. \& Boonsarngsuk, V. Fractured metallic tracheostomy tube: A rare complication of tracheostomy. Respiratory Medicine Case Reports 19, 46-48 (2016).

[10] Herrag, M. et al. Flexible Bronchoscopic Removal of a Fractured Metallic Tracheostomy Tube. Journal of Bronchology \& Interventional Pulmonology 18, 164-167 (2011).

[11] Otto, R. A. \& Davis, W. Tracheostomy tube fracture: an unusual etiology of upper respiratory airway obstruction. The Laryngoscope 95, 980-981 (1985).

[12] Maru, Y. K., Puri, N. D. \& Majid, A. An unusual foreign body in the tracheobronchial tree. J. Laryngol. Otol. 92, 1045-1048 (1978).

[13] Alvi, A. \& Zahtz, G. D. Fracture of a synthetic fenestrated tracheostomy tube: Case report and review of the literature. American Journal of Otolaryngology 15, 63-67 (1994).
[14] Sood, R. K. Fractured tracheostomy tube. J. Laryngol. Otol. 87, 10331034 (1973)

[15] Okafor, B. C. Fracture of tracheostomy tubes: Pathogenesis and prevention. J. Laryngol. Otol. 97, 771-774 (1983).

[16] Tracheostomy tube fatigue. An unusual cause of inhaled foreign body - PubMed. https://pubmed.ncbi.nlm.nih.gov/3998639/.

[17] Fractured silver tracheostomy tube: a case report and literature review - PubMed. https://pubmed.ncbi.nlm.nih.gov/2699679/.

[18] Gupta, S. C. \& Ahluwalia, H. Fractured tracheostomy tube: an overlooked foreign body. J. Laryngol. Otol. 110, 1069-1071 (1996).

[19] Gana, P. N. \& Takwoingi, Y. M. Fractured tracheostomy tubes in the tracheobronchial tree of a child. International Journal of Pediatric Otorhinolaryngology 53, 45-48 (2000).

[20] Fractured Inner Tracheostomy Tube: an Unusual Tracheobronchial Foreign Body | Srinagarind Medical Journal. https://li01.tcithaijo.org/index.php/SRIMEDJ/article/view/14309.

[21] Higuchi, O., Adachi, Y., Ichimaru, T., Asai, M. \& Kawasaki, K. Foreign body aspiration in children: A nationwide survey in Japan. International Journal of Pediatric Otorhinolaryngology 73, 659-661 (2009). 